\title{
Electro-Tactile Feedback System to Enhance Virtual Reality Experience
}

\author{
D. S. Pamungkas and K. Ward
}

\begin{abstract}
Virtual Reality (VR) has been proven to be capable of enabling its users to experience immersive virtual environments and interact with the computer. Most VR applications utilize stereo visual perception, however, stereo visual perception alone is not enough to fully immerse users in VR environments. Haptic feedback technology when applied to VR applications can offer improved immersion and interactivity and can extend the range of sensations experienced by the user. For example, tactile feedback can enable the user to feel an object they touch with their virtual hand or body. To provide tactile feedback in VR applications most researchers construct cumbersome mechanical actuators and linkages for applying feedback forces or vibrations to the user's real hand or body. Having to wear cumbersome feedback hardware can have the negative effect of making the user more aware of their physical body which can reduce the sense of being immersed in the VR world. To overcome this drawback we propose an alternative feedback system which is comprised of a hand gesture interface, a stereo visual head-set and an electro-tactile feedback system. Our electro-tactile feedback system is compact, simple, inexpensive and capable of delivering a variety of feedback sensations to the user. Experimental results are provided which demonstrate how the electro-tactile feedback system can enhance both the sense of immersion and interactivity for VR users.
\end{abstract}

Index Terms-Electro-tactile VR feedback, haptic feedback, immersive.

\section{INTRODUCTION}

Virtual reality (VR) is an emerging technology with increasing application. The main aim of VR is to enable the user to feel immersed in a 3D simulated environment and experience the VR world much like they experience the real world. For this to be possible both the hardware and software has to be devised to enable the user to both see $3 \mathrm{D}$ virtual environment from a first person view and interact with it. Part of this interactivity may involve the user grasping and/or manipulating objects in VR space which has additional challenges.

Most VR researchers, like Sherman and Craig [1], define VR as a computer simulation comprised of a synthetic environment which can be perceived in such a way as to make the user feel immersed in the simulated environment. VR technology can be applied to different applications, the main ones being games for recreational or entertainment purposes or rehabilitation tools for stroke patients. In fact, VR technology can make these applications more enjoyable and effective [2]. Other VR applications include assisting

Manuscript received February 26, 2015; revised August 9, 2015.

Daniel Sutopo Pamungkas and Koren Ward are with the School of Computer Science and Software Engineering, University of Wollongong, Australia (e-mail: dsp572@uowmail.edu.au, koren@uow.edu.au). users to gain certain skills, e.g. training a doctor to do medical surgery [3], or to help people with spinal cord injury in controlling a car [4]. Design work may also be conducted using VR technology, e.g. pipelines design [5]. VR technology can also be useful for helping user to become familiar with working in remote place, e.g. in the deep ocean or the outer space.

Most VR systems offer visual feedback only by using a Head-Mounted Display (HMD) with head pose and position tracking to achieve 3D perception and interactivity with the simulated environment [6], or CAVE (Cave Automatic Virtual Environment) which uses 3D data projectors aimed at walls to immerse the user in the virtual world [7]. When combined with adequate 3D simulator software and processing these systems are able to make virtual objects appear like natural objects by producing in-depth perception of the environment [8].

Several studies have attempted to integrate haptic feedback into VR systems. These studies include using force feedback in a medical simulation to obtain information on virtual body surfaces [9]; a commercial force feedback device to help users to learn Chinese characters [10] and game-related technology in which a system of actuators is integrated to the joystick to give a haptic sensation to the user [11].

However, equipping a VR system with haptic feedback can be complicated to construct, expensive and cumbersome to the user. Furthermore, having to wear cumbersome feedback hardware can have a negative effect by making the user more aware of their physical body which can reduce the sense of being immersed in the VR world.

To overcome these limitations we have been experimenting with applying electro-tactile feedback to VR systems. Electro-tactile feedback involves applying adhesive electrodes or fabric to the skin and stimulating the nerves with a mild electric current. The main benefit of this kind of feedback system is in its simplicity and low cost. Electro-tactile feedback also has more bandwidth compared to vibro-tactile feedback systems which makes it possible to deliver a diverse range of tactile sensations.

In Section II of this paper we provide a brief overview of existing VR haptic feedback systems and applications. Section III outlines the implementation of our electro-tactile feedback system. Section IV describes some VR applications that demonstrate the effectiveness of our proposed feedback system. These applications include virtual hand interactions with: bouncing balls, a camp fire, textured surfaces and gun kickback. Concluding remarks are provided in the Section V.

\section{BACKGROUND}

The key component of any VR system is the display which should deliver the immersive experience to the user with the 
virtual word. There are several approaches to create a 3D visual perception, the main ones being CAVE and HMD, see [6], [7]. The most expensive option, CAVE, requires a room where the walls, floor and ceiling are fully-covered with screens that project the virtual environment. The user also normally wears active shutter 3-D stereo glasses to perceive depth form the projected images. On the other hand, a HMD display is attached to a user's head and supplies 3-D stereo visual information to the user's eyes.

The low cost, compact size and mobility of HMD-based display devices makes HMD preferred over CAVE for most consumer applications. This is despite the difficulty this type of display can impose on the user for controlling other input devices like the keyboard and mouse which occurs mainly because the user cannot see the surrounding real environment when wearing the headset [12].

To overcome this problem, data gloves or a hand gesture interface with tracking, like the Leap Motion [13], can be integrated into the system. Hand tracking and hand gesture interfaces can also facilitate incorporating touch or haptic feedback into the system, providing the hand tracking is not obscured by the feedback device.

Sherman and Craig [1] have mentioned that to produce an immersive and interactive experience for users with the virtual word, a multi-sensory feedback system is required, e.g. vision, sound and haptic feedback. Haptic feedback refers to virtual forces or other tactile information being sent to the sensory neurons in the skin of the user by some means.

Various researchers have made numerous attempts at incorporating haptic and force feedback into VR systems mainly by utilizing vibration and/or force feedback devices.

For example, David et al. [14] have developed a prototype of VR system by using a pair of vibration gloves to deliver haptic information to stroke patient for rehabilitation training purposes. In this system the user wears the gloves in front of tracking cameras which detect hand movements. Each glove has 19 DC motors in its palm and fingers. The motor vibration is activated by a collision between an object and the virtual hand.

In another case, Terziman et al. utilize DC motors to vibrate the floor for simulating the step effects of a giant creature in entertainment applications [15].

Several researchers have utilized force-feedback devices to enrich their VR systems. For example, Våpenstad et al has utilized Xitact - a hand held remote tracking instrument to simulate larascopic surgery [9]. Here, a virtual surgery simulator is controlled and manipulated in response to both vision and force-feedbacks delivered to the user when the virtual tool makes contact with virtual body parts. To achieve the force feedbacks a configuration of pulleys, vacuum actuators, rubber plugs, servomotors and elastic lines are utilized.

A similar force pulley feedback system is used to enhance a VR Spiderman gaming application [16]. Here a virtual feeling of being Spiderman connected to spider web strands is achieved by delivering force feedbacks via actuators and lines to the fingertips together with tracking the user's hand movements with a camera. This is particularly effective when the user moves from one place to another in the VR game by swinging on the virtual web strands as explained in [17]. This haptic feedback device has three DC motors to apply pressure to the fingertips to create the effect of feeling surfaces on virtual objects.

Another novel approach by Blake and Gurocak [18] proposes the utilization of magnetorheological (MR) fluid actuators in a glove to deliver force-feedback for virtualizing grasping sensations within a VR environment. A magnetorheological fluid is a type of smart fluid which is comprised of a carrier fluid, usually oil, filled with iron particles. When exposed to a magnetic field, the fluid greatly increases its viscosity becoming like a viscoelastic solid.

To increase the range of haptic feedback sensations some researchers have attempted to combine force feedback devices with vibration feedback devices. For example, in [19], Hernandez et al. attempt to incorporate force feedback into movable vibration tactors. Here, a force-feedback device, called Omega7, is utilized to deliver feedback information to the user from collisions between the user's virtual hand and virtual objects. The Omega7 can be described as a force feedback handle tiles with $4 \times 4$ tactors to give both a textural feeling of the surfaces of a virtual object as well as any virtual forces applied by them.

Although all the above feedback devices have been demonstrated to be effective in specific VR applications they have the drawback of being rather complex, expensive, application specific and cumbersome to wear; particularly when electro-mechanical actuators and linkages are used to deliver force feedback and/or vibrations to the user.

Bulky input/output devices tend to make the user more conscious of the feedback device which may also detract from achieving a truly immersive experience. Furthermore, most vibration feedback devices (tactors) have greatly limited frequency and amplitude bandwidth, making them incapable of delivering a diverse range of sensations by modulating both the frequency and amplitude of the vibrations.

To overcome these drawbacks we have been experimenting with electrocutaneous or electro-tactile feedback with VR systems. Electro-tactile feedback is a type of haptic feedback which utilizes small electric currents to stimulate nerves in the skin. As this type of feedback system only requires relatively simple circuits and electrodes placed on the skin, it can considerably reduce both the cost and amount of hardware needed to deliver tactile sensations to a user.

Although electro-tactile feedback cannot replicate direct force and other types of touch sensations, it can produce a wide range of tactile sensations without reducing the sensitivity of sensory nerves to the stimulus [20]-[22].

By modulating both the frequency and amplitude of the electric stimulus delivered to the skin we have shown that many real tactile sensations can be approximated with electro-tactile feedback,

In the following section, we provide details of the implementation of our VR electro-tactile feedback system, including a discussion on a number of preliminary experimental results which demonstrate the potentials of this feedback system.

\section{EleCtRo-TACTILE FEEDBACK SySteM}

\section{A. Overview}

The proposed VR feedback system is comprised of an 
Oculus Rift HMD [23] with a Leap Motion hand-tracking unit positioned on the front of a Rift headset, as shown in Fig. 1. The Oculus Rift headset and the Leap Motion unit are connected to a PC and interfaced to applications that were implemented with the Unity game engine [24]. A custom built electro-tactile feedback unit is also connected to the PC and driven by the Unity applications, as depicted in Fig. 2.

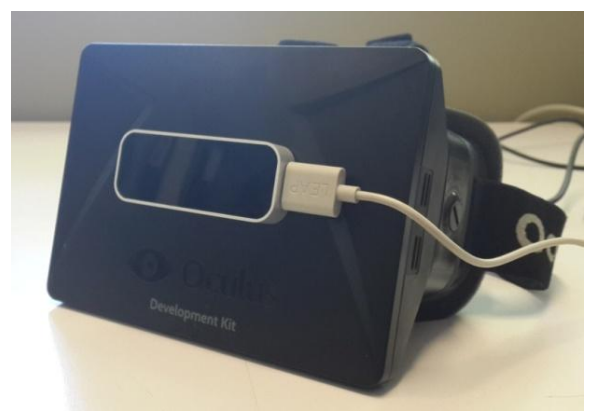

Fig. 1. Oculus rift HMD fitted with a Leap Motion hand tracker.

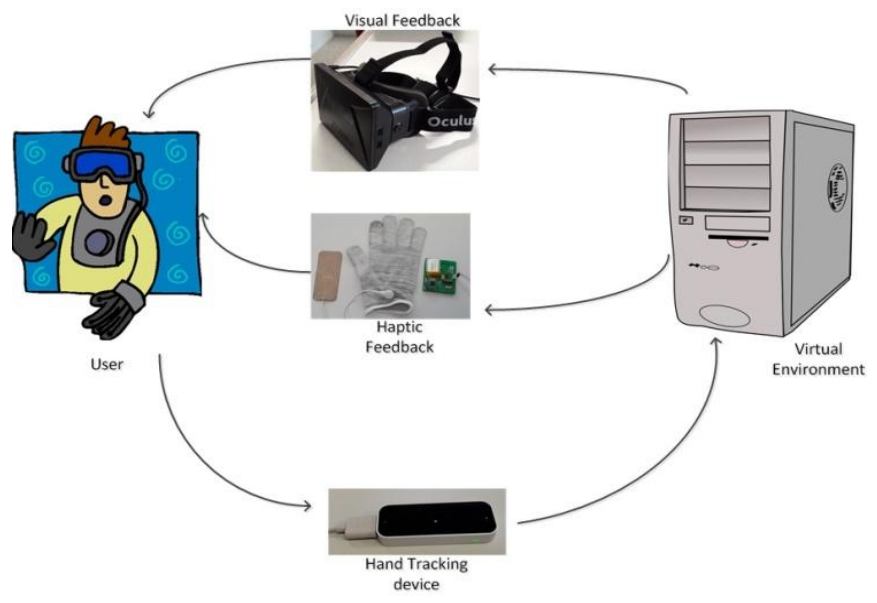

Fig. 2. VR electro-tactile feedback system.

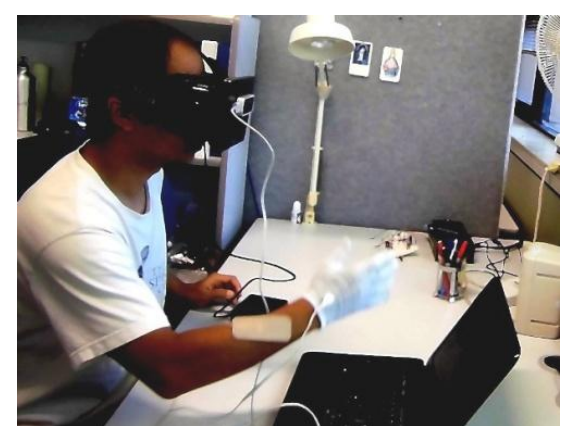

Fig. 3. User operating VR electro-tactile feedback system.

\section{B. Visual Feedback and Hand Control}

The vision feedback and hand control system consists of an Oculus Rift HMD and a Leap Motion unit fitted to the front of the headset, as shown in Fig. 1 and 3. The Oculus Rift HMD has head tracking hardware and is capable in providing color 3D stereoscopic vision to the user with 100-degrees viewing angle. This is somewhat similar to natural vision and enables the operator to feel as if he/she is immersed in the virtual environment.

Leap Motion is a hand tracking device and is comprised of IR LEDs and stereo infrared cameras for both illuminating and detecting the user's hands. It is capable of accurately tracking the user's hands, fingers (and/or a pointing tool) in real time and delivering this information to an application in

\section{skeletal form.}

The Unity game engine software enables the programmer to devise interactive virtual 3-D environments and provide the user with a first-person stereo view of the simulated 3-D environment and the user's virtual hand via the Oculus Rift HMD.

Fig. 2 depicts the flow of information between the main components of our system. Firstly, hand and head tracking information from the Leap Motion and Oculus Rift are sent to the PC running the VR application. From this information, the VR application projects the user's virtual right hand into the virtual environment and determines if any collisions with objects have occurred. The animation software then calculates and delivers to the HMD the user's virtual view of the virtual environment. If any virtual hand touch or collision events with objects have occurred within the virtual environment an appropriate feedback signal is sent to the electro-tactile feedback unit.

\section{Electro-Tactile Feedback}

The electro-tactile feedback system is comprised of wireless USB transmitter unit and a Transcutaneous Electro Neural Stimulation (TENS) receiver unit connected to two TENS electrodes, as shown in Fig. $4 \mathrm{a}$ and $4 \mathrm{~b}$. The TENS electrodes are comprised of a conductive fabric glove and an adhesive patch, as shown in Fig. 4c. By wearing the glove and placing the adhesive TENS electrode on the back of the wrist almost all the stimulus is delivered to the hand.

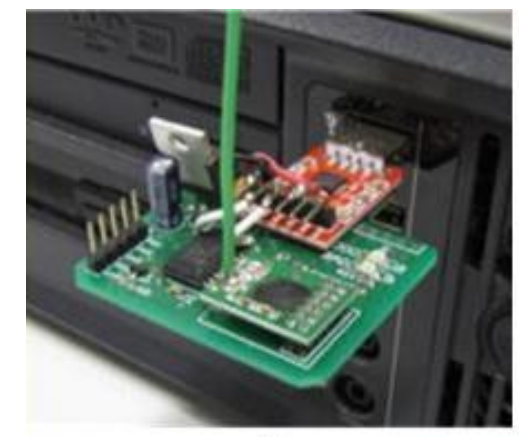

a

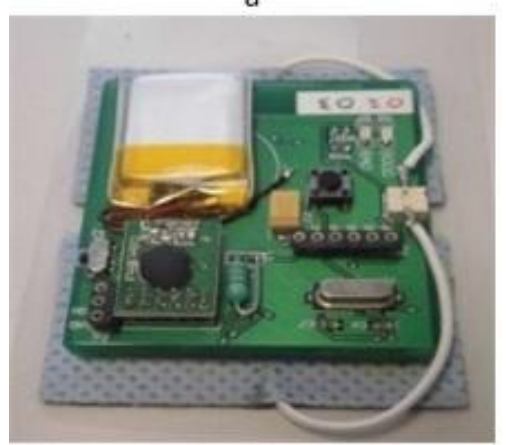

b

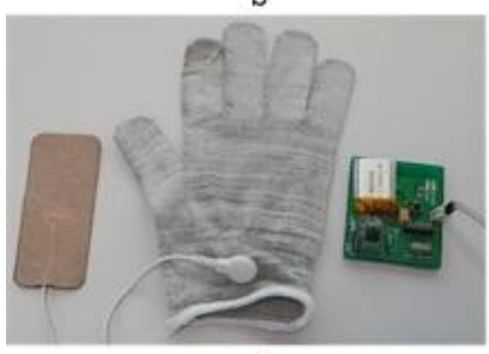

C

Fig. 4. a. Feedback transmitter, b. TENS receiver unit, c. Glove and patch electrodes connected to receiver unit. 
The TENS unit converts the tactile feedback information from the VR application into electric pulses. Fig. 5 shows an oscilloscope trace of a typical TENS pulse. The amplitude can be adjusted between 40-80V and usually preset to suit the user's comfort level. The intensity is controllable by varying the pulse width between $10-100 \mu$ s. The frequency can also be varied between $10-120 \mathrm{~Hz}$.

Tactile feedback sensations are delivered whenever the user's virtual hand or fingers make contact, or intersect with, virtual objects. The stimulus can also be calibrated to suit the user's comfort level. There should be no stimulus when there is no contact between the virtual hand and any virtual objects.

By specifying combinations of frequency and intensity, a range of sensations can be delivered to the user's hand. Generally, we decided to make low intensity mild sensations represent touch, fast high intensity sensations represent impacts and continuous med-high intensity sensations represent warm-hot-burn sensations.

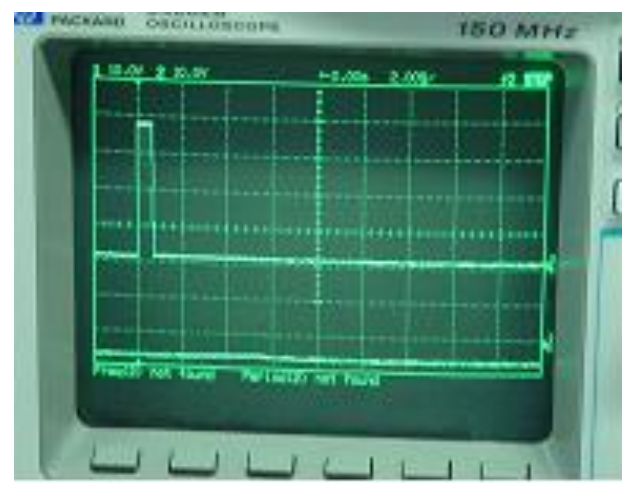

Fig. 5. TENS output waveform

\section{EXPERIMENT METHOD}

To demonstrate the effectiveness of the proposed electro-tactile feedback system, three experiments were performed with three users. These experiments were devised to test the electro-tactile feedback unit's ability to deliver tactile sensations to the user's hand based on VR hand interactions with various virtual objects. All VR applications were implemented with the Unity3D animation software development kit (SDK) within the Unity game engine.

The first experiment involved bouncing a ball in an isolated area by hitting it. The second experiment involved touching walls and feeling their texture. The third experiment involved tactile interactions with a camp fire inside an isolated room. Prior to each experiment each user was asked to set the minimum intensity to zero and the maximum to the highest intensity they could tolerate continuously.

\section{A. Bouncing Ball}

In this experiment three virtual balls (large, medium and small) were setup to bounce easily within a walled isolated virtual environment due to their near perfect elastic collisions with the walls, ceiling and floor. Whenever a ball makes contact with the user's virtual hand tactile stimulus is delivered to the user's hand. The amount of stimulus depends on both the size and speed of the ball. Fig. 6 shows a screenshot of the bouncing balls and user's hand in the virtual environment. The objective is for the user to use his or her hand to hit the balls and make them bounce faster. The user was asked to repeat this task both with and without the tactile feedback switched on and indicate if the tactile feedback improved the VR immersive experience and interactivity. After 5 minutes use each all users reported that the electro-tactile feedback made them feel more immersed in the game, aware when a ball was hit and this improved their concentration on the objective task.

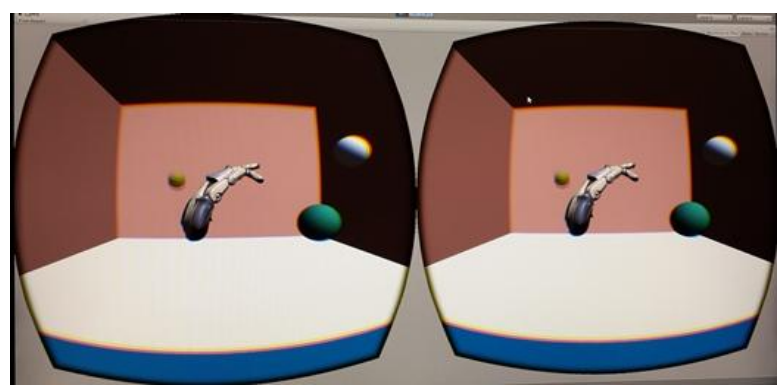

Fig. 6. Hitting bouncing balls in a VR environment.

\section{B. Camp Fire}

The objective of the camp fire experiment was for users to experience temperature changes within a virtual environment via tactile feedback. This involved setting up a simulated fire near the back of the environment and triggering tactile feedback events when the user's virtual hand is near the fire, see Fig. 7. The intensity of the stimulus delivered to the user depends on the distance the hand is from the fire. The maximum intensity occurs when the hand is inside the fire. Users were asked to first feel the warmth from the fire and then to hold their hand in the fire. All users reported that they could feel the heat from the fire. None of the users were able to tolerate holding their hand in the fire for more than a split second.

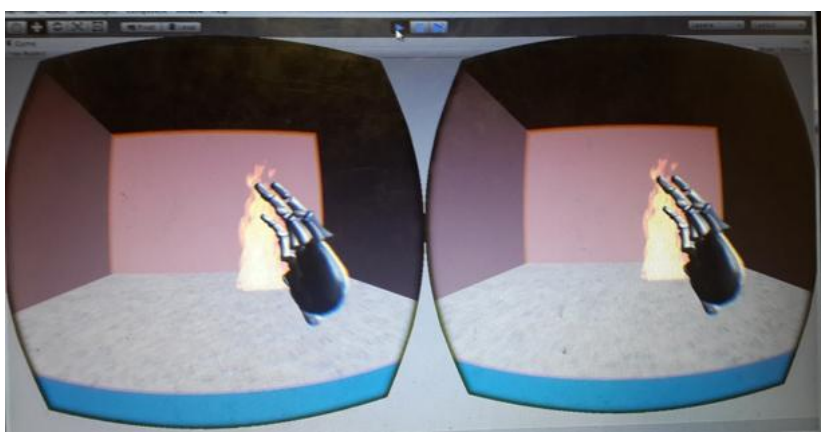

Fig. 7. Feeling a camp fire in a VR environment.

\section{Touching Different Texture}

In this experiment, four different surface textures were rendered on the walls and the floor of a virtual environment, as shown in Fig. 8. Namely: smooth, course brick, bumpy plaster and multi-cavity. Each textured surfaces was mapped to a different tactile feedback stimulus by setting both the frequency and intensity to approximate the texture. Textures were arbitrarily be assigned to walls and the floor prior to the user entering the virtual environment. Users were first asked to familiarize themselves with the different textures on the walls and floor by feeling them with their virtual hand and then exit the room. The textures were then reassigned to different walls and hidden from view by rendering the walls and floor with blank colors. Each user was then asked to 
reenter the environment and identify the walls based on their (hidden) texture. All users were able to correctly classify the walls and floor based on their texture.

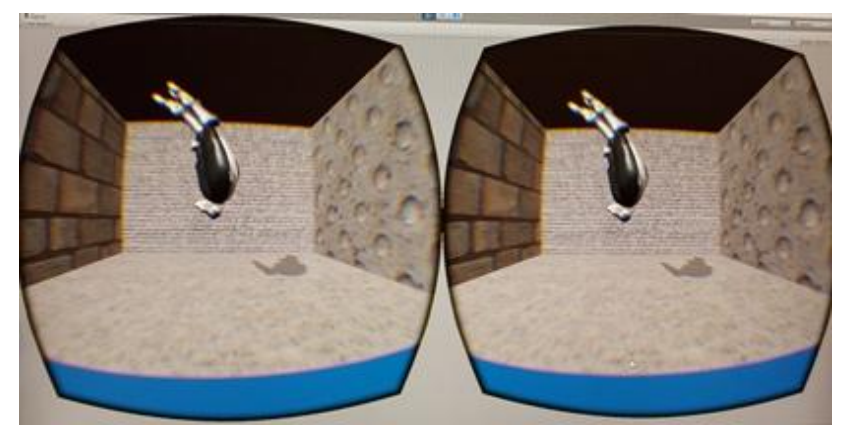

Fig. 8. Touching textured walls within a VR environment.

\section{Boot Camp Gun Kickback Demo}

Boot camp is a third-person demonstration shooting game available in the Unity3D SDK, see Fig. 9. To enhance the interactivity we applied a tactile feedback jolt sensation to the hand when each shot was fired in order to give a gun kickback sensation to the user when the gun was fired. Users were asked to play the game both with and without the tactile kickback turned on. All users reported that the kickback sensation improved immersion and interactivity of the game.

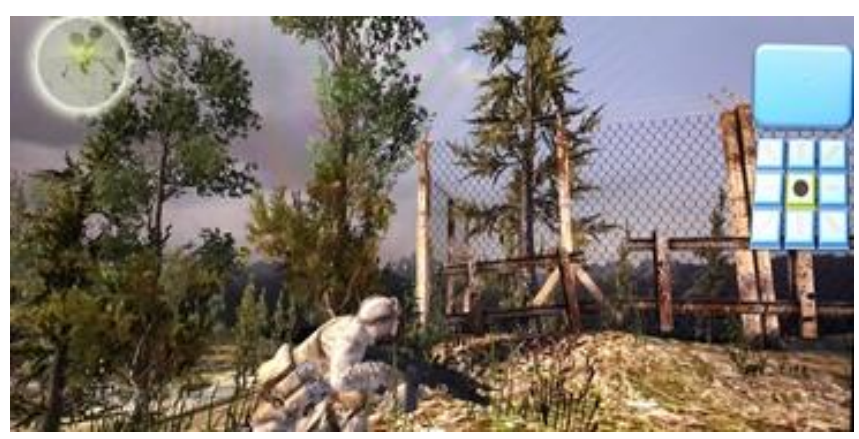

Fig. 9. Shooting a gun with kickback in a VR game.

\section{CONCLUSION}

Haptic feedback technology when applied to VR applications can offer improved immersion and interactivity. However, most existing VR haptic feedback systems are expensive, require the user to wear cumbersome feedback hardware and are limited in the type of haptic sensations they can deliver to the user. To improve on these limitations we have proposed an alternative feedback system based on electro-tactile feedback. Our electro-tactile feedback system is compact, simple, inexpensive and capable of delivering a variety of feedback sensations to the user. Our experimental results demonstrate how electro-tactile feedback can enhance both the sense of immersion and interactivity for VR users.

In our future work, we intend to expand this study by applying electro-tactile feedback to multi-user game applications and tele-presence systems.

\section{REFERENCES}

[1] W. R. Sherman and A. B. Craig, Understanding Virtual Reality: Interface, Application, and Design, $1^{\text {st }}$ ed. San Fransisco, USA: Springer, 2003, ch. 1, p. 1.
[2] K. Laver, S. George, J. Ratcliffe, and M. Crotty, "Virtual reality stroke rehabilitation-hype or hope?" Australian Occupational Therapy Journal, vol. 58, no. 3, pp. 215-219, June 2011.

[3] G. Riva, A. Gaggioli, D. Villani, A. Preziosa, F. Morganti, R. Corsi, G Faletti, and L. Vezzadini, "NeuroVR: An open source virtual reality platform for clinical psychology and behavioral neurosciences," Studies in Health Technology and Informatics, vol. 125, pp. 394-399, 2007.

[4] N. E. Carlozzi, V. Gade, A. Rizo, and D. Tulsky, "Using virtual reality driving simulators in persons with spinal cord injury: Three screen display versus head mounted display," Disability and Rehabilitation: Assistive Technology, vol. 8, no. 2, pp. 176-180, 2013.

[5] M. Karkoub, M. G. Her, and J. M. Chen, "Design of an interactive pipeline inspection VR system," Computer Applications in Engineering Education, vol. 19, no. 4, pp. 814-825, December 2001.

[6] Y. Kim, D. Jo, U. Yang, K. Kim, and G. Lee, "Interactive panoramic VR system to expand spatialness and depth of 3D zone using see-through HMD glasses," in Proc. the 11th ACM SIGGRAPH International Conference on Virtual-Reality Continuum and its Applications in Industry, 2012, pp. 349-349.

[7] F. Grani, F. Argelaguet, M. Badawi, R. Gaugne, S. Serafin, and A. Lecuyer, "Audio-visual attractors for capturing attention to the screen when walking in CAVE systems," in Proc. Virtual Reality (VR), 2014, pp. 75-76.

[8] P. Narayanan, P. Rander, and T. Kanade, "Constructing virtual worlds using dense stereo," in Proc. Sixth International Conference on Computer Vision, 1998, pp. 3-10.

[9] C. Våpenstad, E. Hofstad, T. Lango, R Marvik, and M. Chmarra, "Perceiving haptic feedback in virtual reality simulators," Surgical Endoscopy, vol. 27, no. 7, pp. 2391-2397, August 2013.

[10] A. Hamam, M. Eid, and A. Saddik, "Effect of kinesthetic and tactile haptic feedback on the quality of experience of edutainment applications," Multimedia Tools and Applications, vol. 6, no. 2, pp. 455-472, November 2013.

[11] A. L. Guinan, M. N. Montandon, A. J. Doxon, and W. R. Provancher, "An ungrounded tactile feedback device to portray force and torque-like interactions in virtual environments," in Proc. Virtual Reality (VR), 2014, pp. 171-172.

[12] P. Havig, J. McIntire, and E. Geiselman, "Virtual reality in a cave: limitations and the need for HMDs?" in Proc. SPIE 8041, Head-and Helmet-Mounted Displays XVI: Design and Applications, June 1, 2011.

[13] D. Bachmann, F. Weichert, and G. Rinkenauer, "Evaluation of the leap motion controller as a new contact-free pointing device," Sensors, vol. 15, no. 1, pp. 214-233, 2015.

[14] D. Sadihov, B. Migge, R. Gasset, and Y. Kim, "Prototype of a VR upper-limb rehabilitation system enhanced with motion-based tactile feedback," in Proc World Haptics Conference (WHC), 2013, pp. 449-454.

[15] D. Terziman, M. Marchal, F. Multon, and A. Lécuyer, "The king-kong effects: Improving sensation of walking in VR with visual and tactile vibrations at each Step," in Proc. IEEE Symposium on 3D User Interfaces, 2012, pp. 19-26.

[16] K. Ishibashi, T. D. Luz, R. Eynard, N, Kita, N. Jiang, H. Segi, K. Terada, K. Fujita, and K. Miyata, "Spider hero: a VR application using pulling force feedback system," in Proc. the 8th International Conference on Virtual Reality Continuum and Its Applications in Industry. 2009, pp. 197-202.

[17] L. Meli, S. Scheggi, C. Pacchiorroti, and D. Prattichizzo, "Wearable haptics and hand tracking via an RGB-D camera for immersive tactile experiences," ACM SIGGRAPH 2014 Posters, Vancouver, Canada, 2014.

[18] J. Blake and H. B. Gurocak, "Haptic glove with MR brakes for virtual reality," IEEE/ASME Transactions on Mechatronics, vol. 14, no. 5, pp. 606-615, October 2009.

[19] N. Garcia-Hernandez, I. Sarakoglou, N. Tsagarakis, and D. Caldwell, "Exploration of virtual surface features with a high performance tactile and force feedback interface," in Proc. IEEE International Conference on Systems, Man, and Cybernetics (SMC), 2012, pp. 2998-3003.

[20] D. Pamungkas and K. Ward, "Tele-operation of A Robot Arm with Electro Tactile Feedback," in Proc IEEE/ASME International Conference on Advanced Intelligent Mechatronics (AIM), 2013, pp. 704-709.

[21] H. Kajimoto, M. Inami, N. Kawakami, and S. Tachi, "SmartTouch-augmentation of skin sensation with electrocutaneous display," in Proc. 11th Symposium on Haptic Interfaces for Virtual Environment and Teleoperator Systems, 2003, pp. 40-46.

[22] M. Peruzzini, M. Germani, and M. Mengoni, "Electro-Tactile Device for Texture Simulation," in Proc IEEE/ASME International 
Conference on Mechatronics and Embedded Systems and Applications (MESA), 2012, pp. 178-183.

[23] I. Goradia, J. Doshi, and L. Kurup, "A review paper on oculus rift \& project Morpheus," International Journal of Current Engineering and Technology, vol. 4, no. 5, pp. 3196-3200, October 2014.

[24] J. H. Bae and A. E. Kim, "Design and development of unity3d game engine-based smart SNG," International Journal of Multimedia and Ubiquitous Engineering, vol. 9, no. 8, pp. 261-266, 2014.

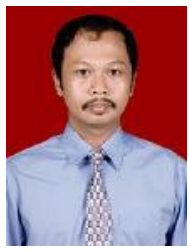

Daniel Sutopo Pamungkas was born in Cirebon, Indonesia on November 28,1975 . He received a bachelor degree in physics engineering from the Bandung Institute of Technology in 1999. He received his masters degree in electronic engineering from the Bandung Institute of Technology in 2007. He is currently enrolled in a PhD degree in computer science at the University of Wollongong, Australia.

His research interests include immersive technology, haptic feedback, virtual reality, robotics, tele-operation. sensors and embedded systems.

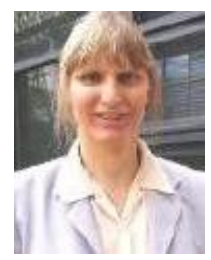

Koren Ward is a researcher and senior lecturer in the University of Wollongong (UOW), Australia. She was born in Wollongong Australia and received her $\mathrm{PhD}$ in computer science from the University of Wollongong in 2001. Her research interests include robotics, sensors, user interfaces, virtual reality, electro-tactile feedback, hand and body gesture extraction, face recognition, gaze tracking, head pose tracking, VR, immersive technologies, tele-presence, adaptive robot control systems, artificial intelligence, genetic algorithms, neural networks, fuzzy logic, intelligent data analysis, data mining and robotic trading systems. She has received a number of large grants from the Australian Research Council to develop assisted technologies for the blind involving tactile feedback. This research work mostly involved the development of human computer interfaces for the blind and indoor and outdoor sensor assisted perception and navigation systems for the blind. The current focus of her research work involves the development of immersive technologies with electro-tactile feedback. 